\title{
Carcinoma epidermoide de canal anal estádio IV: complicações clínicas de doença avançada
}

\author{
Epidermoid carcinoma of anal canal stage IV: clinical complications of \\ advanced disease
}

\author{
FERNANDA BELLOTTI FORMIGA ${ }^{1}$; ALESSANDRA VICENTINI CREDIDIO²; DALTRO LEMOS ROSA ${ }^{3}$; \\ JOSÉ CÉSAR ASSEF ${ }^{4}$; CHIA BIN FANG ${ }^{5}$; PERETZ CAPELHUCHNIK ${ }^{6}$; WILMAR ARTUR KLUG7
}

\begin{abstract}
${ }^{1}$ Pós-graduanda de Coloproctologia da Santa Casa de São Paulo - São Paulo (SP), Brasil; ${ }^{2}$ Residente de Coloproctologia da Santa Casa de São Paulo - São Paulo (SP), Brasil; ${ }^{3}$ Pós-graduando de Coloproctologia da Santa Casa de São Paulo - São Paulo (SP), Brasil; ${ }^{4}$ Professor Adjunto da Faculdade de Ciências Médicas da Santa Casa de São Paulo e Chefe do Serviço de Emergência da Irmandade da Santa Casa de São Paulo - São Paulo (SP), Brasil; ${ }^{5}$ Professor Adjunto da Faculdade de Ciências Médicas da Santa Casa de São Paulo - São Paulo (SP), Brasil; ${ }^{6}$ Professor Titular da Faculdade de Ciências Médicas da Santa Casa de São Paulo - São Paulo (SP), Brasil; ${ }^{7}$ Professor Titular da Faculdade de Ciências Médicas da Santa Casa de São Paulo - São Paulo (SP), Brasil.
\end{abstract}

FORMIGA FB, CREDIDIO AV, ROSA DL, ASSEF JC, FANG CB, CAPELHUCHNIK P, KLUG WA. Carcinoma epidermoide de canal anal estádio iv: complicações clínicas de doença avançada. Rev bras Coloproct, 2011;30(4): $\quad$-454.

RESUMO: O carcinoma anal é uma entidade rara que representa 4\% dos tumores malignos da região anorretal, dentro os quais o carcinoma epidermoide constitui o tipo histológico mais comum. É relatado o caso de um paciente masculino, 54 anos, com carcinoma epidermoide de canal anal localmente avançado e com metástases ósseas no diagnóstico, feito após complicação infecciosa local com repercussão sistêmica. Descrevemos a evolução do paciente após o diagnóstico da neoplasia e as dificuldades de manejo clínico encontradas neste caso que são secundárias às complicações inerentes à doença de base.

Descritores: canal anal, diagnóstico, neoplasias do ânus, complicações, estadiamento de neoplasias.

\section{INTRODUÇÃO}

O carcinoma anal é uma entidade rara que representa $4 \%$ dos tumores malignos da região anorretal ${ }^{1} \mathrm{e}$ 1 a $2 \%$ de todos os tumores do trato gastrointestinal ${ }^{2}$.

O carcinoma epidermoide é o tipo histológico mais comum dentre as neoplasias do canal anal, responsável por aproximadamente $85 \%$ das lesões malignas dessa região ${ }^{3}$. Atualmente, tem sido observada uma modificação na epidemiologia dessa doença em decorrência do aumento da população de homens jovens homossexuais infectados pelo vírus da imunodeficiência humana $(\mathrm{HIV})^{4-6}$, porém ainda se observa um predomínio em mulheres entre a sexta e sétima décadas de vida ${ }^{7,8}$.

\section{RELATO DO CASO}

J.A.S., 54 anos, sexo masculino, negro. Paciente deu entrada no Serviço de Emergência apresentando lesões fétidas e dolorosas no glúteo, bolsa escrotal e coxa esquerda. A lesão inicial, localizada na coxa, havia surgido há cinco anos e estava sendo tratada como furunculose, com recorrência frequente. Relatava que a lesão havia progredido em extensão nos últimos dois meses, associada à dor, incontinência fecal, perda ponderal de $10 \mathrm{~kg}$ e dificuldade de mobilização da perna esquerda.

Ao exame, apresentava-se taquicárdico, desidratado, descorado, com linfonodomegalia inguinal bila-

Disciplina de Coloproctologia da Santa casa de São Paulo.

$\overline{\text { Recebido em 15/03/2010 }}$

Aceito para publicação em 09/04/2010 
teral (um linfonodo endurecido e fixo de $1,5 \mathrm{~cm}$ de diâmetro à esquerda) e dor à palpação de crista ilíaca esquerda. O exame proctológico evidenciava lesões vegetantes ulceradas, infectadas, com fibrina e saída de secreção purulenta nas nádegas, face posterior da coxa esquerda e bolsa escrotal, além do ânus entreaberto. Ao toque, palpação e anuscopia, notava-se enduramento da nádega esquerda e região perianal, lesão circunferencial esbranquiçada e endurecida em todo canal anal, esfíncter hipotônico e reto sem alterações.

Os exames laboratoriais evidenciavam leucocitose com desvio à esquerda e insuficiência renal. Indicado desbridamento cirúrgico amplo, após compensação clínica inicial (Figuras 1 e 2). O material resultante

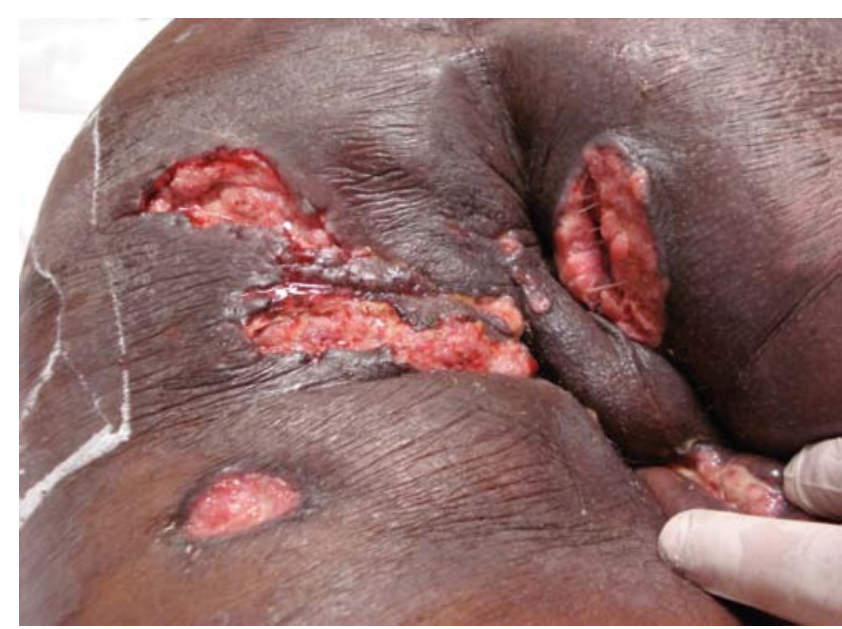

Figura 1 - Aspecto das lesões após desbridamento cirúrgico.

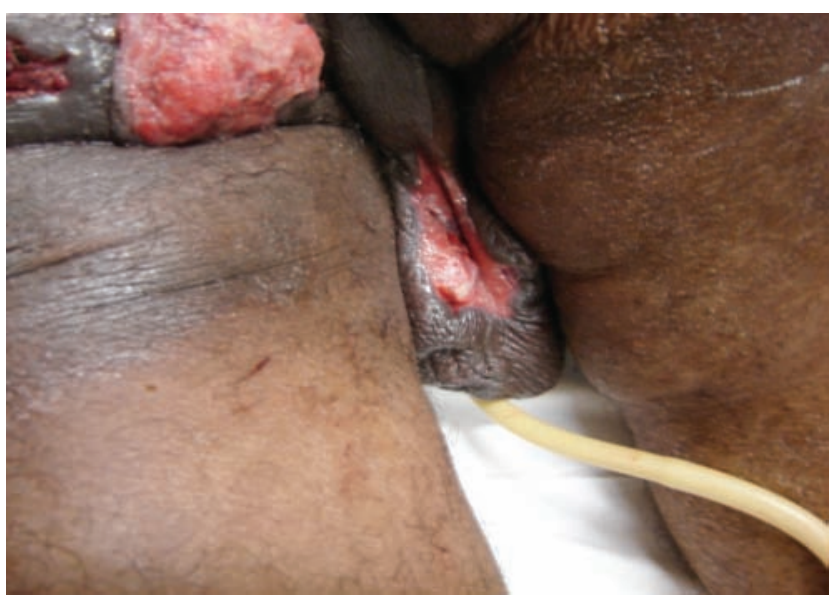

Figura 2 - Extensão para bolsa escrotal. do desbridamento foi enviado para estudo histológico, que revelou carcinoma espinocelular invasivo de canal anal. As sorologias para hepatites, sífilis e HIV foram não-reagentes.

Após 21 dias de antibioticoterapia, diagnosticouse uma coleção na região glútea por meio de tomografia computadorizada da pelve (Figura 3), a qual foi puncionada (Figura 4).

Exames complementares para estádio do tumor foram realizados, sendo evidenciada destruição do complexo esfincteriano pela Ultrassonografia anorretal radial bidimensional (Figuras 5 e 6 ) e metástases ósseas em ambas as cabeças femorais e ossos ilíacos pela Ressonância Nuclear Magnética (Figura 7). Tomografia Computadorizada de tórax e abdome não evidenciaram metástases hepáticas ou pulmonares. Biópsia excisional do linfonodo inguinal esquerdo também foi realizada, evidenciando metástase linfonodal de carcinoma espinocelular.

Paciente evoluiu com piora do estado geral, bexigoma de repetição por provável invasão da inervação pélvica, desnutrição, má aceitação da dieta, dificuldade de mobilização por dor em membro inferior esquerdo e hipercalcemia refratária secundária às metástases ósseas, necessitando de sondagem vesical de demora e nutrição enteral. Após 14 dias de novo esquema de antibioticoterapia contra Staphylococcus aureus e Pseudomonas aeruginosa multiresistentes isolados na secreção colhida após a punção da região glútea, paciente melhorou do processo infeccioso local, porém

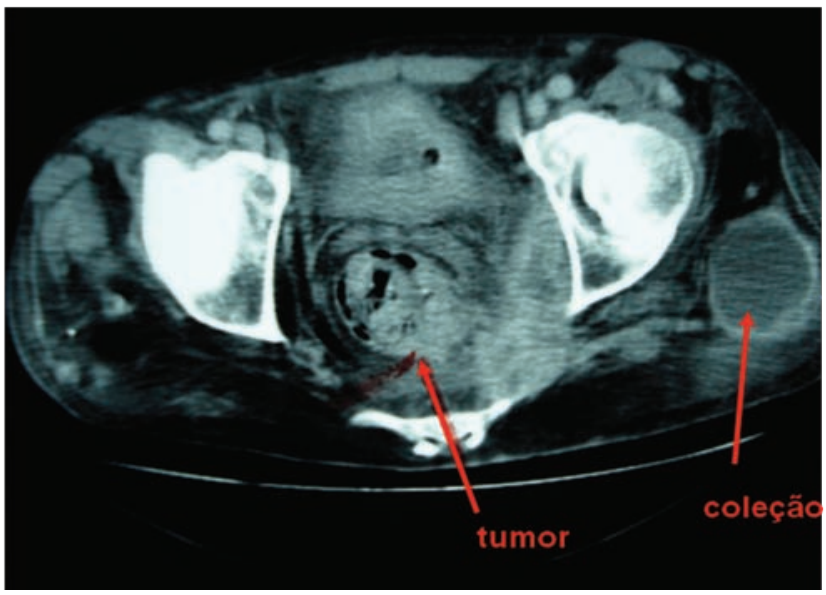

Figura 3 - Tomografia computadorizada evidenciando tumor e coleção glútea. 


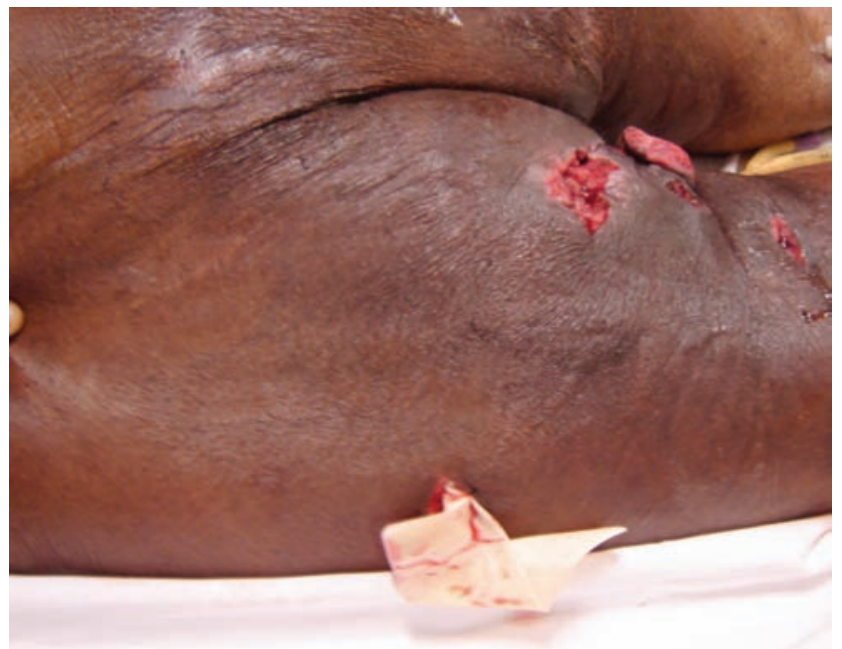

Figura 4 - Coleção puncionada e drenada.

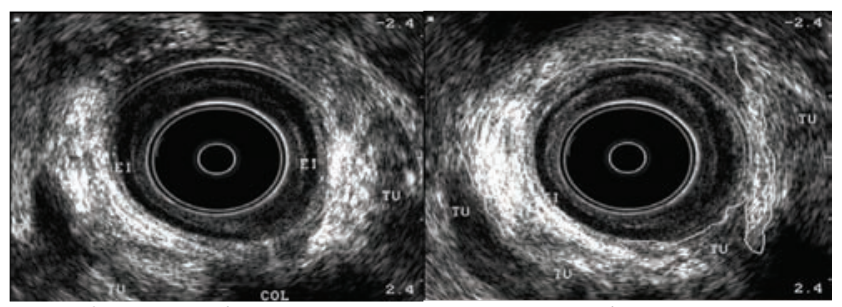

EI: esfíncter anal interno; TU: tumor; COL: coleção.

Figura 5 - Ultrassonografia anorretal do canal anal.

evoluiu com pneumonia nosocomial, associada à empiema pleural e óbito após 45 dias de internação.

\section{DISCUSSÃO}

A apresentação clínica do tumor de canal anal é muito inespecífica e, naa grande maioria dos casos, se confunde com condições anorretais benignas, uma vez que ambas coexistem em aproximadamente $50 \%$ dos pacientes $^{9}$. Os principais sintomas são dor (60\%), sangramento (59\%), sensação de tumoração ocupando o canal anal (25-30\%) e prurido (25\%).

Pacientes que apresentam doença avançada ao diagnóstico podem referir diminuição do calibre das fezes, constipação, incontinência fecal secundária ao envolvimento da musculatura esfincteriana, emagrecimento e linfonodomegalia inguinal ${ }^{13}$, estando os três últimos sintomas presentes no caso relatado. A associação entre os sintomas inespecíficos e a falta de sus-

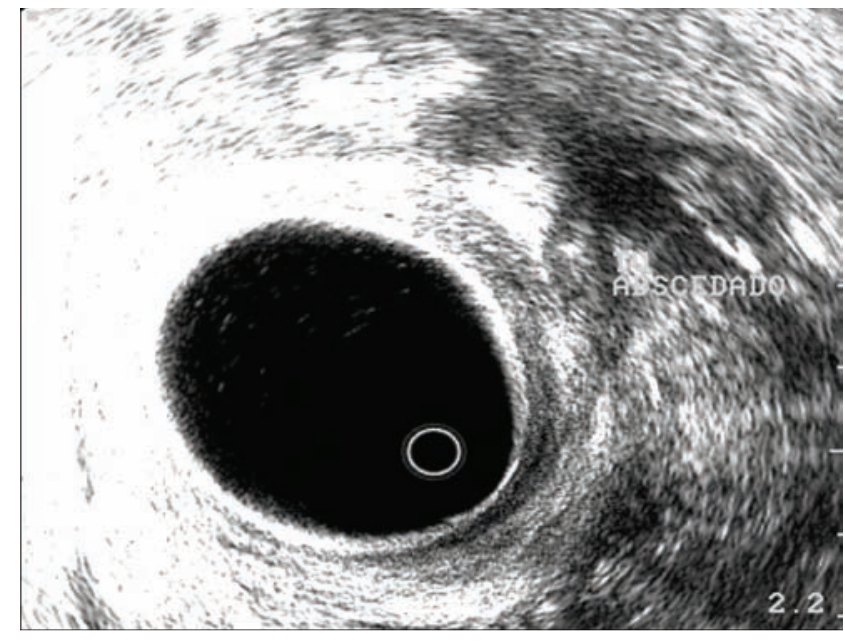

Figura 6 - Ultrassonografia anorretal do reto: tumor abscedado.

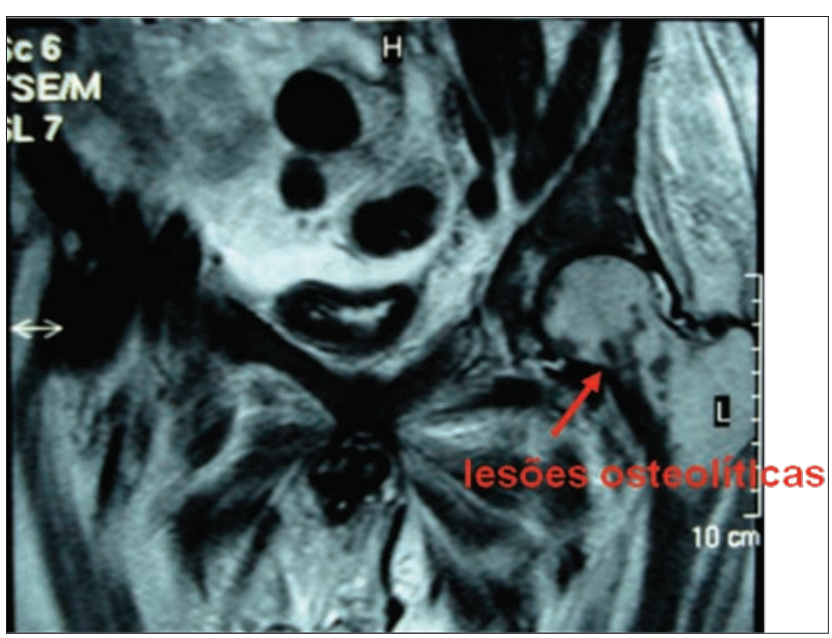

Figura 7 - Ressonância nuclear magnética evidenciando lesões líticas na cabeça do fêmur (metástase óssea).

peita clínica resulta em 30 a 50\% de casos com doença localmente avançada no momento do diagnóstico ${ }^{13}$.

O tumor de canal anal está associado a comportamento local agressivo e sua disseminação ocorre principalmente de forma locorregional, com invasão de estruturas vizinhas (bexiga, vagina, uretra) e através da via linfática, com comprometimento de linfonodos das cadeias inguinal e pélvica (perirretal e ilíaca interna). A incidência de linfonodos inguinais clinicamente positivos nos tumores T3 ou T4 é de 30 a $60 \%{ }^{14}$. 
Os achados clínicos do paciente em questão condizem com os dados encontrados na literatura, pois ele já apresentava, no momento do diagnóstico, neoplasia localmente avançada (T4) associada a metástase óssea e linfonodomegalia inguinal bilateral (N3).

O tumor de canal anal raramente cursa com metástases a distância, e corresponde a menos de $10 \%$ dos casos. As metástases são mais comumente encontradas no fígado e no pulmão ${ }^{15,16}$, com eventual disseminação óssea.

Aproximadamente 60 a $70 \%$ dos pacientes são incluídos nos estádios I ou $\mathrm{II}^{17,18}$. A taxa de sobrevida em cinco anos para pacientes com tumores T1 ou T2 é de $86 \%$, enquanto para pacientes com tumores T3 ou T4 é de 60 e $45 \%$, respectivamente ${ }^{19}$.

O tratamento do carcinoma epidermoide de canal anal sofreu importante modificação nas últimas décadas e, atualmente, é baseado em quimioterapia e radioterapia combinadas, no esquema de $\mathrm{Nigro}^{20}$, como substituição ao procedimento cirúrgico ${ }^{16,21}$. O procedimento cirúrgico baseado na amputação abdominoperineal é indicado como tratamento de resgate nos casos que apresentaram falha à terapia combinada (QT-RT) ou nos casos de recidiva local do tumor ${ }^{22}$. Porém, essas modalidades de tratamento não se aplicam aos casos de doença disseminada, reservando-se para esta condição apenas a realização de quimioterapia paliativa ${ }^{23}$.

Conforme observado no caso relatado, a falta de condições clínicas do paciente, secundária à disseminação da doença tanto localmente quanto a distância, dificulta e posterga o início do tratamento quimioterápico, mesmo paliativo.

O carcinoma epidermoide estádio IV e localmente avançado pode determinar dificuldades na manipulação clínica do paciente, uma vez que não há proposta terapêutica curativa e as intercorrências clínicas e alterações na qualidade de vida são inevitáveis.

ABSTRACT: Anal carcinoma is a rare entity that represents $4 \%$ of anorectal malignant tumors, and the squamous cell carcinoma is the most common histological type. We report the case of 54-year-old male patient with locally advanced squamous cell carcinoma of the anal canal and metastatic bone disease at diagnosis, which was made after local infectious complications with systemic impact. We describe the evolution of the patient after the diagnosis and the difficulties of clinical management that are secondary to the complications related to the underlying disease.

Key words: anal canal, diagnosis, anus neoplasms, complications, neoplasm staging.

\section{REFERÊNCIAS}

1. Beahrs OH, Wilson SM. Carcinoma of the anus. Ann Surg 1976;184(8):422-8.

2. Greenlee RT, Murray T, Bolden S, Wingo PA. Cancer statistics. CA Cancer S Clin 2000;50(1):7-33.

3. Stearns MW, Urmacher C, Sternberg SS. Cancer of the anal canal. Curr Probl Cancer 1980;4(12):1-44.

4. Ryan DP, Campton CC, Mayer RJ. Carcinoma of the anal canal. N Engl J Med 2000;342(11):798-800.

5. Modesto VL, Gottesman L. Doenças sexualmente transmitidas e manifestações anais da AIDS. Clin Cir Am Norte 1994;74:1501-34.

6. Chauvenic L, Buthaud X, Falcou MC, Mosseri V, De la Rochefordière, Pierga JY et al. Anal canal cancer treatment: practical limitations of routine prescriptions of concurrent chemotherapy and radiotherapy. $\mathrm{Br} \mathrm{J}$ Cancer 2003;89(11):2057-61.

7. Moscicki AB, Hills NK, Shiboski S, Darragh TM, Jay N, Towell $\mathrm{K}$ et al. Risk factors for abnormal anal cytology in young heterosexual women. Cancer Epidemiol Biomarkers Prev 1999;8(2):173-8.
8. Gervaz P, Allal AS, Villinger P, Bühler L, Morel P. Squamous cell carcinoma of the anus: another sexually transmitted disease. Swiss Med Wkly 2003;133(25-26):353-9.

9. Greenall MJ, Quan SH, Decoss J. Epidermoid cancer of the anus. Br J Surg 1985;72 Suppl:S97-103.

10. Singh R, Nime F, Mittelman A. Malignant epithelial tumors of the anal canal. Cancer 1981;48(2):411-5.

11. Schraut WH, Wang CH, Dawson PJ, Block GE. Depth of invasion, location and size of cancer of the anus dictate operative treatment. Cancer 1983;51(7):1291-6.

12. Tanum G, Tveit K, Carlsen KO. Diagnosis of anal carcinoma - doctor's finger still the best. Oncology 1991;48(5):383-6.

13. Deans GT, McAleer JJA, Spence RAJ. Malignant anal tumors. Br J Surg 1994;81(4):500-8.

14. Noffsinger A, Witte D, Fenoglio-Preiser CM. The relationship of human papillomaviruses to anorectal neoplasia. Cancer 1992;70(5 Suppl):1276-87.

15. Minsky BD, Hoffman JP, Kelsen DP. Cancer of the anal region. In: DeVita Jr VT, Hellman S, Rosenberg SA, editors. Cancer: principles and practice of oncology. $6^{\text {th }}$ edition. Philadelphia: Lippincott, Williams and Wilkins; 2001. p. 1319-42. 
16. Esiashvili N, Landry J, Matthews RH. Carcinoma of the anus: strategies in management. Oncologist 2002;7(3):188-99.

17. Gerard JP, Chapet O, Samiei F, Morignat E, Isaac S, Paulin $\mathrm{C}$ et al. Management of ingutinal lymph node metastases in patients with carcinoma of the anal canal: experience in a series of 270 patients treated in Lyon and review of the literature. Cancer 2001;92(1):77-84.

18. Boman BM, Moertel CG, O’Connell MJ, Scott M, Weiland LH, Beart RW et al. Carcinoma of the anal canal: A clinical and pathologic study of 188 cases. Cancer 1984;54(1):114-25.

19. Touboul E, Schlienger M, Buffat L, Lefkopoulos D, Pène F, Parc R et al. Epidermoid carcinoma of the anal canal. Results of curative - intent radiation therapy in a series of 270 patients. Cancer 1994;73(6):1569-79.

20. Nigro N, Vaitkevicius V, Considine S. Combined therapy for cancer of the anal canal: a preliminary report. Dis Colon Rectum 1974;17(3):354-6.
21. Fleshner PR, Chalasani S, Chang GJ, Levien DH, Hyman $\mathrm{NH}$, Buie WD et al. Practice parameters for anal squamous neoplasms. Dis Colon Rectum 2008;51(1):2-9.

22. Renehan AG, Saunders MP, Schofield PF, O'Dwyer ST. Patterns of local disease failure and outcome after salvage surgery in patients with anal cancer. Br J Surg 2005;92(5):60514.

23. Rousseau Jr DL, Thomas Jr CR, Petrelli NJ Kahlenberg MS. Squamous cell carcinoma of the anal canal. Surg Oncol 2005;14(3):121-32.

\section{Endereço para correspondência:}

Fernanda Bellotti Formiga

Avenida Líbero Badaró, 1.208 - Jardim São Caetano CEP 09581-610 - São Caetano do Sul (SP), Brasil

Fax: (11) 4238-0638

E-mail: fformiga@colband.com.br 